\title{
Betamethasone 17-valerate by aerosol in childhood asthma
}

\author{
JANNA F. FREARS, LYN C. WILSON, and MAX FRIEDMAN \\ From Whittington Hospital. Dartmouth Park Hill, London, and the Clinical Research Unit, Glaxo Laboratories Ltd., \\ Greenford, Essex
}

Frears, J. F., Wilson, L. C., and Friedman, M. (1973). Archives of Disease in Childhood, 48, 856. Betamethasone 17-valerate by aerosol in childhood asthma. A double-blind cross-over trial of betamethasone 17-valerate (B17V) administered by aerosol was carried out by alternating two placebo periods with two treated periods at monthly intervals in 20 children with severe asthma. During the trial, the average of twice-daily peak expiratory flow readings was significantly increased in every child during actively treated months, compared with placebo months. Every child had fewer symptoms while using $B 17 \mathrm{~V}$, and the average reduction for the group as a whole was $71 \%$. Very much less additional medication for asthma was required during months of active treatment. The observed clinical response to therapy was dramatic in over half the children. No side effects were noted. Under the conditions of this study, we conclude that B17V by aerosol appears to be safe, and is effective for the control of asthma in children.

The efficacy of orally administered steroid preparations for the control of asthma is such that they would be universally used were it not for their undesirable side-effects when administered in pharmacological doses. These include manifestations of Cushing's syndrome, suppression of the pituitary-adrenal axis, and stunting of growth in children. The unwillingness of physicians to drop such valuable therapy has led to a search for a means of retaining pharmacological properties, while reducing either the dose or the side-effects of steroid compounds: for example, by using an alternative method of administration.

There is a theoretical advantage in delivering the drug direct to the bronchial tree, where it might be expected to exert a local action at much lower doses than are needed orally. Early work with inhaled hydrocortisone and prednisolone powders (Brockbank and Pengelly, 1958; Herxheimer, McAllen, and Williams, 1958) and with metered aerosol (Franklin et al., 1958) showed results which seemed encouraging, but proved disappointing because of the high dosage required. Dexamethasone by aerosol in adults (Fisch and Grater, 1962; Arbesman, Bonstein, and Reisman, 1963) and in children (Siegel et al., 1964; Kravis and Lecks,

Received 2 May 1973.
1966) proved to be an effective method of controlling asthma, but the drug was absorbed in amounts sufficient to give systemic effects.

New interest has been aroused in this field since it has become apparent that steroids with a high index of topical activity, but relatively low adrenal suppressive action, may prove both clinically effective and systemically harmless. The compounds betamethasone 17-valerate (B17V) and beclomethasone dipropionate fall into this group. Work with the latter (Lal et al., 1972; Brown, Storey, and George, 1972; Clark, 1972; Choo-Kang et al., 1972) has indicated a good clinical response, though the interpretation of pituitary-adrenal function tests carried out on patients using the aerosol has been complicated by the fact that many of them had been previously maintained on longterm oral steroids at suppressive doses.

Previous experience of B17V has been limited mainly to the treatment of skin conditions (as Betnovate). Absorption through the skin in amounts sufficient to cause adrenal suppression has occurred, but only when $30 \mathrm{~g}$ /day was applied to $60 \%$ of the body surface under polyethylene occlusion in patients with acute dermatoses (James, Munro, and Feiwel, 1967). When used as a retention enema in the treatment of distal proctocolitis, $5 \mathrm{mg} \mathrm{B} 17 \mathrm{~V}$ daily was found to be as 
effective as $20 \mathrm{mg}$ prednisolone 21-phosphate, and caused less suppression (Multicentre Trial, 1971). As a nasal spray it is effective for allergic rhinitis (Czarny and Brostoff, 1968). 1 patient out of 12 using $0.4 \mathrm{mg} \mathrm{B17V}$ intranasally for 7 days showed evidence of adrenal suppression.

The aim of the present trial was to show that B17V is effective in the control of chronic or frequently recurrent asthma. In this notoriously capricious disease, where subjective observations are inevitably influenced by placebo response and spontaneous remissions, assessment can only be properly made through a double-blind cross-over study. An attempt was made to assess the effect on the pituitary-adrenal axis by serial cortisol estimations throughout the trial.

\section{Method}

Subjects. 22 children were selected from the Whittington Hospital paediatric asthma clinic on the following criteria. (a) Their symptoms conformed with the definition of asthma by Scadding (1966) and they were poorly controlled on their current therapy. (b) They had not received ACTH or oral steroid preparations for 6 weeks before starting the trial. (c) They were able, after tuition, to use a pressurized aerosol properly. (d) The child's mother was judged able and willing to co-operate in daily assessments at home and frequent attendance in outpatients.

Materials and protocol. A 4-month trial period was planned, during which active and placebo treatments were alternated at monthly intervals as active/placebo/ active/placebo or placebo/active/placebo/active. Children were allocated to each sequence by a random preselection not known to the patient or physician.

Metered aerosols delivering $100 \mu \mathrm{g} \mathrm{B17V}$ per burst in the fluohydrocarbon propellants Arcton 11 and 12 were used. Placebo aerosols identical in appearance contained the same propellants. Each child was allotted a numbered box containing prepacked aerosols in pairs, each pair representing a month's supply and labelled for the appropriate month.

During a run-in period of approximately 2 weeks, parents and children were trained in the use of a pressurized aerosol and in the method of record keeping described by Connolly and Godfrey (1970) which was continued throughout the trial. On a diary card points were given to match observed symptoms of asthma up to a daily maximum of 13 , the patient scoring nil when symptom free. Morning and evening peak flow readings (best of 3 on each occasion) were recorded on the same card, as well as any drug usage in addition to the trial aerosol.

At the outset of the trial, in order to reassure the patient when symptoms flared up on placebo therapy, it was explained that the aerosols would contain two different drugs, one of which might be more effective than the other. Patients did not know the periodicity of the sequence of aerosols, which were renewed once a fortnight. Mothers were told that the drug would act by preventing the child from getting asthma, and not by immediate relief of symptoms, and were reassured that every additional medication required to control the child's symptoms would be given throughout the trial period. They were instructed to continue the medication the child was having before the trial. No attempt was made to wean the patient off any other drugs unless the asthma chart showed a score of 0 for at least 4 days previously, and then maintenance medication was discontinued one drug at a time over the course of the next few days. Disodium cromoglycate (DSCG) (Intal) was the last drug to be discontinued. When symptoms recurred, all the previous medication with the exception of DSCG was re-introduced until another clear 4-day period occurred.

During the first month of the trial patients were seen weekly, and thereafter at fortnightly intervals. At each attendance height, weight, forced expiratory volume at one second $\left(\mathrm{FEV}_{1}\right)$, and peak expiratory flow rate (PEF) were recorded, and a subjective assessment of clinical state was made by parent and physician on a basis of same/better/worse than the period before the last visit. Blood for plasma cortisol was taken at fortnightly intervals wherever possible and an estimation was made by the fluorimetric method of Mattingly (1962). At the end of the trial, the patient's mother and the physician recorded their impressions of each month in turn.

\section{Results}

The results of 20 of the 22 children who were started in this trial are presented. One patient failed to attend regularly, and the other was withdrawn from the trial because she became so ill in a placebo month that the code was broken to see whether she had benefited from active treatment.

The 20 children whose results were analysed included 13 boys and 7 girls with a mean age of 8 years 7 months (range 4 years 6 months-14 years 11 months). 11 patients received $B 17 \mathrm{~V}$ and 9 received placebo in the first trial month. The results of 2 representative patients are presented in Fig. 1 and 2.

In view of the variable nature of the disease, it has been recently pointed out (Silverman et al., 1972) that occasional measurements of lung function are not of much value in assessing therapeutic responses in asthma. Subjective comments by the parent and physician made in the clinic are usually unreliable. The results of the trial have been assessed on the basis of the information obtained from diary entries made at home, and the observations made at the periodic visits to the asthma clinic, with the emphasis on the former.

\section{Observations made in the home.}

Changes in PEF readings. Values were obtained for twice-daily peak flow readings over the 4 trial 


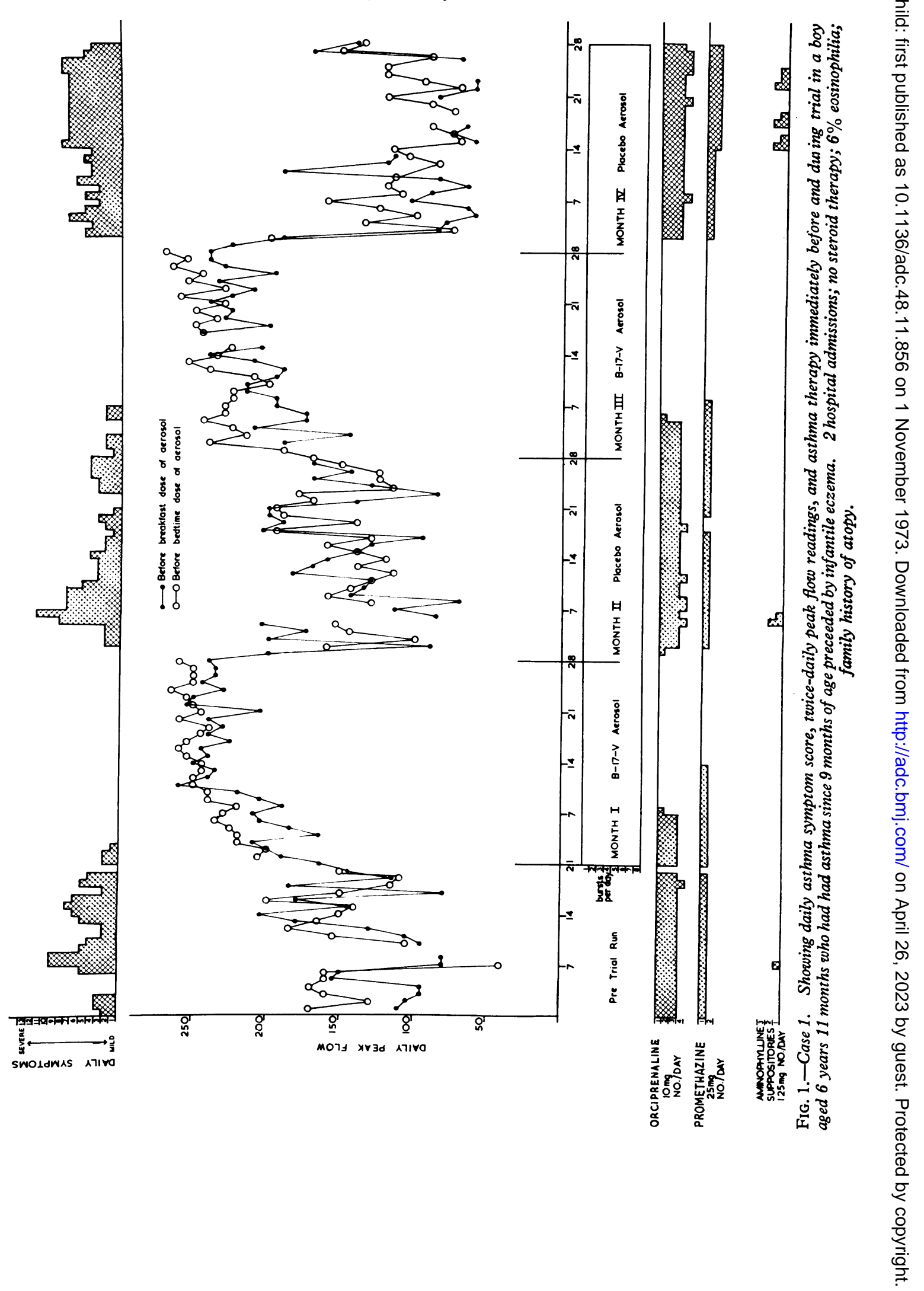



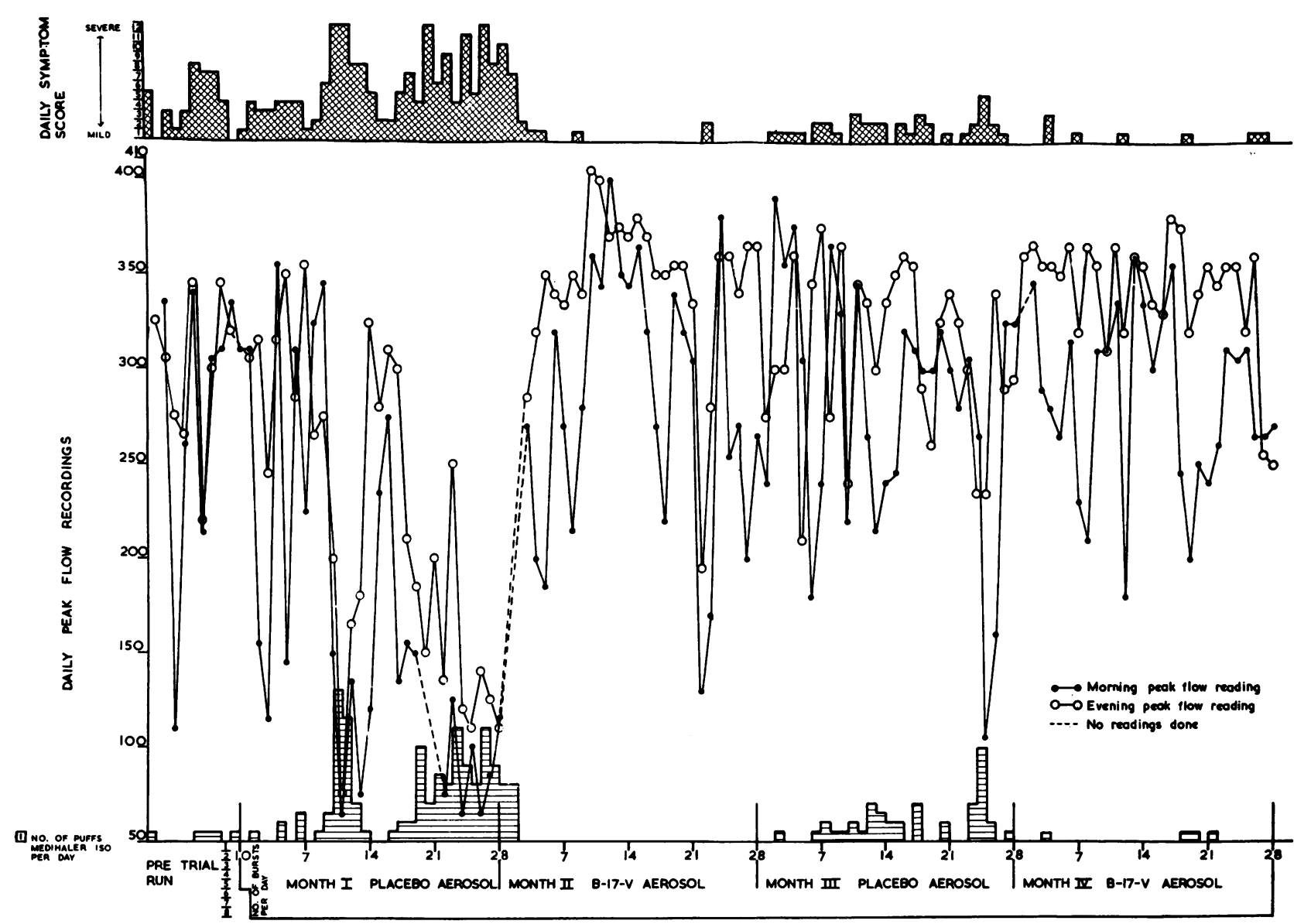

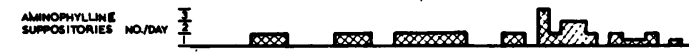

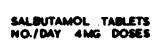

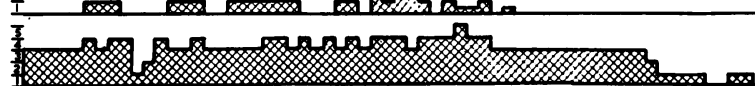

\&

FIG. 2. -Case 12. Shou ing daily asthma symptom score, twice-daily peak flow readings, and asthma therapy immediately before and during trial in a boy aged 11 years 5 months who had had asthma since 6 years of age. Hay fever; no hospital admissions or steroid therapy. Chest deformity; 28\% eosinophilia; family history of atopy; addicted to medihaler-iso aerosol, taking 50 doses overnight in last bad attack.

months in 19 out of 20 children, 1 patient being considered too young to use a peak flow meter properly.

When the average twice-daily readings for the two 28-day placebo periods was compared with the average of the two 28-day treated periods (Table I and Fig. 3), each child showed an increase. This ranged from $3 \%$ to $85 \%$, with a mean of $31 \%$. Compared with the average over the pretrial run, which was carried out over a shorter time span (16 days average, range 7-27), peak flow readings during the treated period were again higher. 9 children averaged above their lowest predicted normal value ( -2 SD) (Weng and Levison, 1969) during active treatment. In 3 cases, readings done before the trial started were higher than during the placebo period, reflecting the variability of symptoms in asthma. In the remaining 16 of the 19 children, the pretrial average was comparable to, or lower than, the placebo average. 
TABLE I

Changes in peak expiratory flow (PEF) readings in 19 cases

\begin{tabular}{|c|c|c|c|c|c|}
\hline Case no. & $\begin{array}{c}\text { Age on entering } \\
\text { trial } \\
(\mathrm{yr})(\mathrm{mth})\end{array}$ & $\begin{array}{c}\text { Lowest } \\
(-2 \text { SD }) \\
\text { normal PEF }\end{array}$ & $\begin{array}{l}\text { Average during } 2 \\
\text { placebo months }\end{array}$ & $\begin{array}{l}\text { Average during } 2 \mathrm{~B} 17 \mathrm{~V} \\
\text { months }\end{array}$ & $\begin{array}{l}\text { Average } \% \text { increase } \\
\text { during } B 17 \mathrm{~V} \text { month }\end{array}$ \\
\hline $\begin{array}{r}1 \\
2 \\
3 \\
5 \\
6 \\
7 \\
8 \\
9 \\
10 \\
11 \\
12 \\
13 \\
14 \\
15 \\
17 \\
18 \\
20 \\
21 \\
22\end{array}$ & $\begin{array}{rr}6 & 11 \\
7 & 4 \\
9 & 10 \\
8 & \\
7 & 9 \\
9 & 2 \\
5 & 10 \\
9 & 2 \\
9 & 7 \\
8 & 11 \\
11 & 5 \\
6 & 7 \\
10 & 11 \\
14 & 11 \\
6 & 11 \\
6 & 4 \\
7 & 10 \\
11 & 2 \\
8 & 2\end{array}$ & $\begin{array}{l}175 \\
140 \\
250 \\
180 \\
200 \\
250 \\
150 \\
225 \\
220 \\
190 \\
285 \\
170 \\
245 \\
300 \\
165 \\
140 \\
180 \\
280 \\
225\end{array}$ & $\begin{array}{c}124 \\
103 \\
165 \\
104 \\
231 \\
233 \\
148 \\
130 \\
152 \\
128 \\
244 \\
145 \\
111 \\
231 \\
125 \\
120 \\
208 \\
165 \\
174 \\
\text { Mean } 160 \cdot 1 \\
\text { SEM } 10 \cdot 9\end{array}$ & $\begin{array}{c}229 \\
162 \\
234 \\
121 \\
242 \\
299 \\
166 \\
218 \\
196 \\
182 \\
314 \\
149 \\
119 \\
349 \\
162 \\
130 \\
119 \\
214 \\
212 \\
\text { Mean } 206 \cdot 2 \\
\text { SEM } 14 \cdot 7\end{array}$ & $\begin{array}{c}85 \\
59 \\
45 \\
17 \\
5 \\
28 \\
12 \\
68 \\
29 \\
43 \\
29 \\
3 \\
7 \\
73 \\
30 \\
8 \\
5 \\
30 \\
22 \\
\text { Mean } 31 \cdot 2 \\
\text { SEM } 4 \cdot 1\end{array}$ \\
\hline
\end{tabular}

B17V, betamethasone 17-valerate.

Paired ' $t$ ' test on differences of averages $P<0.001$.

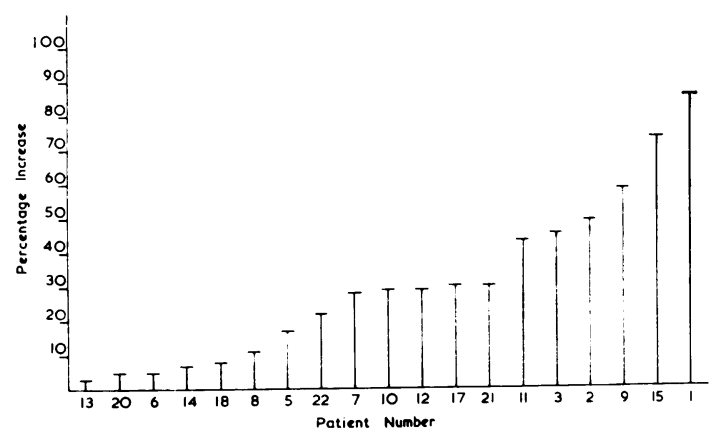

FIG. 3.-Percentage increase in peak expiratory flow readings during months on betamethasone 17-valerate compared with placebo months.

Changes in symptom score. Every patient had a lower average monthly symptom score during treatment with B17V than during the placebo period (Fig. 4). The total average monthly score for all the children during the placebo period was 64, and during the equivalent treated period it fell to 19 . This represents a $71 \%$ reduction in symptoms with the B17V aerosol.

Changes in additional drug usage. 19 of the 20 children were on continuous medication with bronchodilators, and some were on additional night sedation, at the outset of the trial. Many of them were able to do without any medication, apart from the trial aerosol, after the first few days of each actively treated month. An attempt was made to assess the variations in drug usage additional to $\mathrm{B} 17 \mathrm{~V}$ by expressing the bronchodilator requirements of children on maintenance therapy as a percentage of their pretrial intake (Table II). In

\section{TABLE II}

Bronchodilator usage during last 7 days of each trial month, expressed as \% of total usage during pretrial run

\begin{tabular}{c|c|c|c|c}
\hline Case no. & $\begin{array}{c}\text { 1st placebo } \\
\text { period }\end{array}$ & $\begin{array}{c}\text { 1st B17V } \\
\text { period }\end{array}$ & $\begin{array}{c}\text { 2nd placebo } \\
\text { period }\end{array}$ & $\begin{array}{c}\text { 2nd B17V } \\
\text { period }\end{array}$ \\
\hline 1 & 100 & 0 & 114 & 0 \\
2 & 42 & 0 & 100 & 100 \\
3 & 200 & 100 & 70 & 66 \\
5 & 0 & 0 & 57 & 0 \\
6 & 100 & 0 & 10 & 50 \\
7 & 10 & 0 & 0 & 0 \\
9 & 112 & 0 & 20 & 0 \\
10 & 48 & 0 & 50 & 0 \\
11 & 110 & 0 & 125 & 0 \\
12 & 127 & 10 & 40 & 20 \\
13 & 100 & 72 & 100 & 100 \\
14 & 63 & 63 & 98 & 32 \\
15 & 100 & 20 & 50 & 0 \\
16 & 100 & 25 & 50 & 0 \\
17 & 33 & 33 & 80 & 0 \\
18 & 100 & 100 & 0 & 0 \\
20 & 100 & 0 & 100 & 0 \\
21 & 200 & 200 & 150 & 150 \\
22 & 150 & 5 & 150 & 150 \\
\hline Average & $94 \cdot 5$ & 33 & 72 & 35 \\
& & & & \\
\hline
\end{tabular}

B17V, betamethasone 17-valerate. 


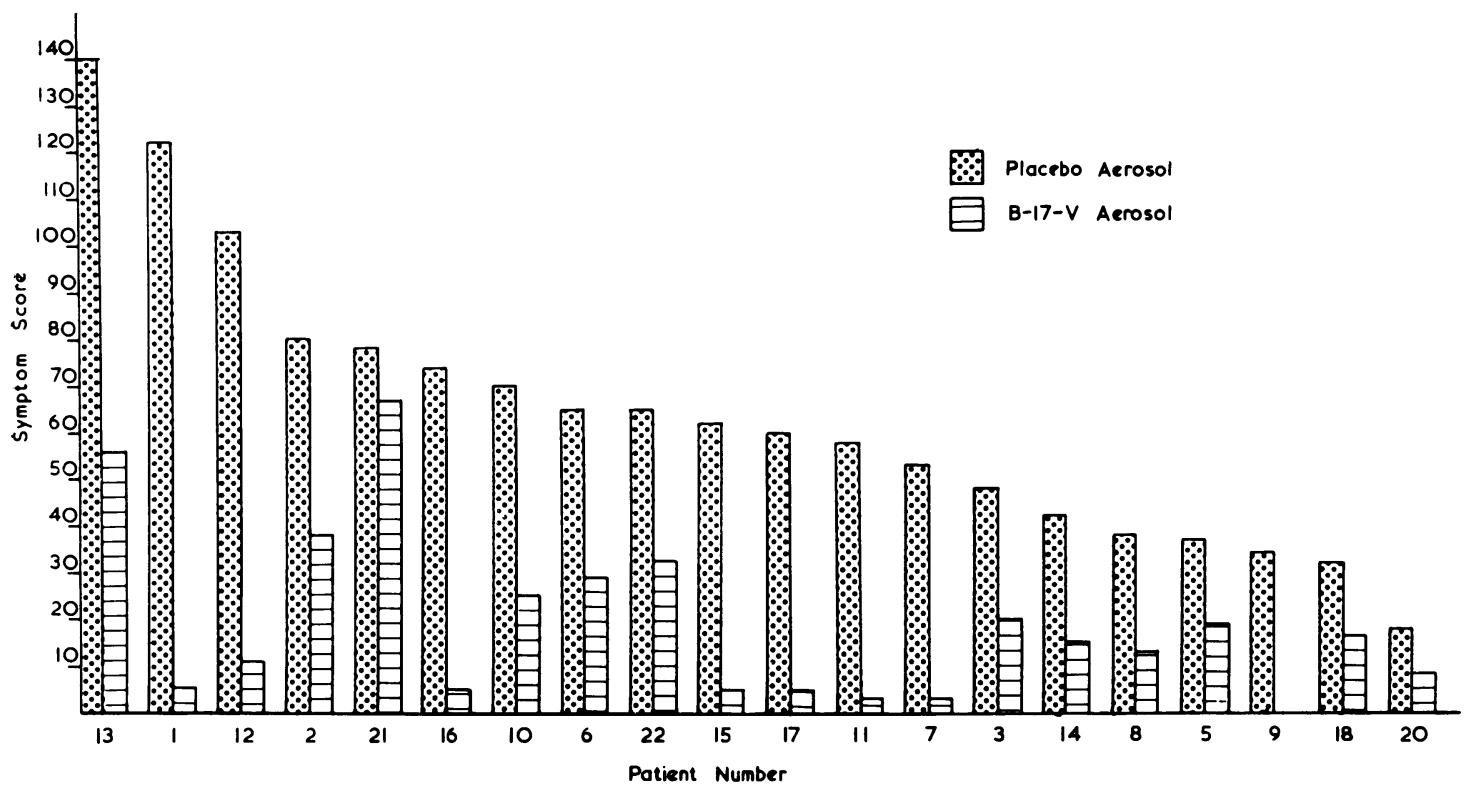

Fig. 4.-Average monthly symptom scores during 2 placebo months compared with 2 months on betamethasone 17-valerate. Average monthly symptom score on active treatment was $18 \cdot 8$ (SEM 4.1), whereas average monthly symptom score in placebo months was $64 \cdot 0$ (SEM 6.8), the difference being significant $(P<0 \cdot 001)$.

order to exclude any carry-over effect from the previous month, the periods selected for comparison were the last 7 days of each trial month. Average bronchodilator intake on placebo was $83 \%$ of pretrial requirements, and on $\mathrm{B} 17 \mathrm{~V}$ it fell to $34 \%$.

Of the 8 children on DSCG at the start of the trial, 7 had discontinued it by the end. Only 1 child appeared to be worse after stopping it.

Two children were using pressurized bronchodilator aerosols for relief of symptoms before the trial, and 1 was dangerously addicted, so that in a recent severe attack he had taken 50 doses overnight. 1 of these 2 did not use the bronchodilator aerosol at all in the active months of the trial, and the addict was only taking very occasional doses (Fig. 2) as opposed to heavy usage in placebo periods. Both children handed in their bronchodilator aerosols shortly after the end of the trial, when they were maintained on long-term B17V.

Over the 4-month period, 121 aminophylline suppositories were used by 13 children, 110 in placebo months and 11 during B17V months, mainly in the first 3 days before the drug had achieved maximum effect. Two children required short courses of prednisolone during placebo months.

Observations made in the asthma clinic. Measurements of $\mathrm{FEV}_{1}$ were made at each attendance on 19 of 20 patients who were able to use a vitalograph. 15 of the 19 had their highest values while on B17V treatment and 14 had their lowest readings while on placebo treatment. Monthly averages were usually higher during treated periods.

Clinical observations usually followed the pattern of the trial, and by the end the response was so clear in 11 cases that the physician was able to make a confident correct guess about the arrangement of months. 11 mothers volunteered a remark to the effect that their child had never been so well since the onset of asthma, and always in reference to a treated period. The remaining 9 children had a less clear-cut response, but the tentative guess made by the physician about the order of the trial was nevertheless always correct.

Serial cortisol estmations. Taking a normal level as $5 \mu \mathrm{g} / 100 \mathrm{ml}$ or above, 6 children (Cases 1, 2, 3, 15, $16,20)$ had low levels at some time during active treatment (Table III). However, in 4 of these patients the levels were subsequently normal while still on active treatment. In 2 patients in whom low values were obtained, only a single estimation was made. 2 patients (Cases 3 and 15) had had previous steroid therapy, which may account for the low values. In 2 (Cases 2 and 15) an intravenous insulin tolerance test and tetracosactrin test (Wood et al., 
TABLE III

Plasma cortisol levels $(\mu \mathrm{g} / 100 \mathrm{ml})$

\begin{tabular}{c|l|l}
\hline Case no. & During B17V months & During placebo months \\
\cline { 1 - 2 } 1 & $5,4,5$ & 11,12 \\
2 & $5,3,5,10$ & $9,5,5,5,11$ \\
3 & 3 & 7,9 \\
5 & $9,13,8,10$ & $7,17,9,8,6$ \\
6 & $7,8,7,8$ & $7,9,8,10,7$ \\
7 & $18,17,20$ & $11,16,9,24,14$ \\
8 & 10,11 & 8 \\
9 & 13,19 & 19,20 \\
10 & $8,9,7,6$ & $6,11,7,11$ \\
11 & $8,6,7,6$ & $23,6,19$ \\
12 & $6,7,7$ & $5,7,10,13,6$ \\
13 & 7 & 6,6 \\
14 & 9 & $10,10,9,6$ \\
15 & 6,4 & $9,5,7$ \\
16 & 4 & 7 \\
17 & 12 & $9,9,15$ \\
18 & 29 & 11 \\
20 & $5,4,9$ & 6,7 \\
21 & 6,9 & $10,7,6$ \\
22 & $8,5,12,6$ & $10,12,11,10$ \\
& &
\end{tabular}

1965), carried out after the trial when they were maintained on long-term $\mathrm{B} 17 \mathrm{~V}$, gave values within the normal range.

\section{Discussion}

The results presented indicate a good clinical response in terms of daily assessment of symptoms, improvement in lung function, and reduction in additional drug usage in all 20 children in the trial.

Some children with milder asthma tended to have a smaller percentage increase in peak flow averages but, on the other hand, their score cards reflected an impressive diminution of symptoms during $\mathrm{B} 17 \mathrm{~V}$ months. Assessment by scoring may be entirely subjective, but it is at least consistent for each patient and reflects the aspect of the disease which is important to him. Peak flow readings alone are not universally reliable for assessment, as one child who scored persistently very reduced readings, in spite of subjective and observed clinical improvement, was subsequently found to be a poor performer with a peak flow meter.

Examination of the data indicates that there is no substantial difference between the results of those children who were given active treatment in the first month of the trial, and those whose first month was a placebo period. A placebo response may account for the observation that 1 or 2 children had worse asthma during the pretrial run than during placebo trial months. This may, however, merely reflect the variability of the disease and emphasize the need for a double-blind trial. No child had worse asthma on active treatment than in either pretrial or placebo periods.

It is interesting to note that there was a reduction in the total symptom score and the bronchodilator usage in the second placebo month, which agrees with the subjective impression of the physician that the children did not have such severe symptoms again after an intervening period of treatment with B17V. There is no evidence of a carry-over effect of B17V other than the 2- to 3-day period clearly shown in Fig. 1.

No factors came to light in judging who will respond to this form of therapy, and it appeared that the age of the child was irrelevant provided he was old enough to use a pressurized aerosol, which in practice turned out to be above 6 years, with the exception of the youngest child aged 4 years 6 months whose mother, herself an aerosol user, was able to hold the aerosol and time the administration correctly. The children who responded least well tended to be those with disturbed family relationships, one important factor possibly being lack of supervision over medication.

The majority of children in this group came from atopic families and suffered from early onset asthma, usually preceeded by eczema. Many had had multiple hospital admissions. One had in the past required maintenance therapy with prednisone for 3 years, and 3 others had had intermittent courses of ACTH and/or oral steroids. 19 were currently on routine conventional therapy with or without DSCG. These children all had fairly severe asthma interfering with their daily lives, and were on the whole candidates for oral steroid or ACTH maintenance treatment. Some of them showed the nutritional effects of chronic asthma, which tended to improve during the trial.

No clinical side effects of excess glucocorticoid activity were observed during the trial, and from results of plasma cortisols so far it is uncertain whether there is suppression of the pituitary-adrenal axis. It seems unlikely, since we have administered $1600 \mu$ g daily to adult volunteers for 7 days and have found no evidence of suppression of the hypothalamic-pituitary-adrenal axis (J. F. Frears and M. Friedman, unpublished data). The total dose administered by aerosol would, if given orally, be insufficient to cause suppression (Friedman et al., 1967).

We thank Miss M. F. Crowley for estimating plasma cortisol levels during the trial.

\section{REFERENCES}

Arbesman, C. E., Bonstein, H. S., and Reisman, R. E. (1963). Dexamethasone aerosol therapy for bronchial asthma. fournal of Allergy, 34, 354.

Brockbank, W., and Pengelly, C. D. R. (1958). Chronic asthma treated with powder inhalations of hydrocortisone and prednisolone. Lancet, 1, 187. 
Brown, H. M., Storey, G., and George, W. H. S. (1972). Beclomethasone dipropionate: a new steroid aerosol for the treatment of allergic asthma. British Medical fournal, 1, 585.

Choo-Kang, Y. F. J., Cooper, E. J., Tribe, A. E., and Grant, I. W. B. (1972). Beclomethasone dipropionate by inhalation in the treatment of airways obstruction. British fournal of Diseases of the Chest, 66, 101.

Clark, T. J. H. (1972). Effect of beclomethasone dipropionate delivered by aerosol in patients with asthma. Lancet, 1, 1361 .

Connolly, N. M., and Godfrey, S. (1970). Assessment of the child with asthma. Fournal of Asthma Research, 8, 31 .

Czarny, D., and Brostoff, J. (1968). Effect of intranasal betamethasone-17-valerate on perennial rhinitis and adrenal function. Lancet, $2,188$.

Fisch, B. R., and Grater, W. C. (1962). Dexamethasone aerosol in respiratory tract disease. Fournal of New Drugs, 2, 298.

Franklin, W., Lowell, F. C., Michelson, A. L., and Schiller, I. W. (1958). Aerosolized steroids in bronchial asthma. Fournal of Allergy, 29, 214.

Friedman, M., Fletcher, J., Hinton, J. M., Lennard-Jones, J. E., Misiewicz, J. J., and Parrish, J. A. (1967). Observations on the absorption of oral betamethasone 17-valerate and its therapeutic value in ulcerative colitis. British Medical fournal, 1, 335.

Herxheimer, H., McAllen, M. K., and Williams, D. A. (1958). Local treatment of bronchial asthma with hydrocortisone powder. British Medical fournal, 2, 762.

James, V. H. T., Munro, D. D., and Feiwel, M. (1967). Pituitaryadrenal function after occlusive topical therapy with betamethasone-17-valerate. L ancet, 2, 1059

Kravis, L. P., and Lecks, H. I. (1966). Inhalation vs oral steroid therapy in childhood asthma. American fournal of Diseases of Children, 111, 343.

Lal, S., Harris, D. M., Bhalla, K. K., Singhal, S. N., and Butler, A. G. (1972). Comparison of beclomethasone dipropionate aerosol and prednisolone in reversible airways obstruction. British Medical fournal, 3, 314.

Mattingly, D. (1962). A simple fluorimetric method for the estimation of free 11-hydroxycorticoids in human plasma. Fournal of Clinical Pathology, 15, 374.

Multicentre Trial (1971). Betamethasone 17-valerate and prednisolone 21-phosphate retention enemata in proctocolitis. British Medical fournal, 3, 84.

Scadding, J. G. (1966). Patterns of respiratory insufficiency. Lancet, 1, 701.

Siegel, S. C., Heimlich, E. M., Richards, W., and Kelley, V. C. (1964). Adrenal function in allergy. IV. Effect of dexamethasone aerosols in asthmatic children. Pediatrics, 33, 245.

Silverman, M., Connolly, N. M., Balfour-Lynn, L., and Godfrey, S. (1972). Long-term trial of disodium cromoglycate and isoprenaline in children with asthma. British Medical fournal, $3,378$.

Weng, T.-R., and Levison, H. (1969). Standards of pulmonary function in children. American Review of Respiratory Diseases, 99, 879 .

Wood, J. B., Frankland, A. W., James, V. H. T., and Landon, J. (1965) A rapid test of adrenocortical function Lancet, 1 , 243.

Correspondence to Dr. M. Friedman, Whittington Hospital, Dartmouth Park Hill, London N19 5JG. 